\title{
Ki67 Proliferative Index in Carcinoid Tumors Involving Ovary
}

\author{
Xiaotun Zhang ${ }^{1} \cdot$ Andrea Jones $^{1} \cdot$ Sarah M. Jenkins ${ }^{2} \cdot$ Yajue Huang $^{1}$ \\ Published online: 18 January 2018 \\ (C) The Author(s) 2018. This article is an open access publication
}

\begin{abstract}
Primary ovarian carcinoid tumors are rare neoplasms that constitute less than $0.1 \%$ of all ovarian carcinomas. However, carcinoid tumors metastatic to ovaries are more common. Cell proliferative rate is an important factor in the determination of neuroendocrine tumor prognosis. Limited data are available as regards Ki67 proliferation index in predicting the physiological features of carcinoid tumors involving the ovary. Pathology files of Mayo Clinic Rochester (1995-2014) were searched, and clinical information was collected from medical records. All cases were stained with an antibody against Ki67, and digital analysis was performed with digital imaging analysis. A total of 36 cases (median age 64 years, range 33-83 years), including 9 primary (median age 68 years, range 33-73 years) and 27 metastatic carcinoid cases (median age 64 years, range 36-83 years), were investigated in the current study. Seven out of nine (77.8\%) primary ovarian carcinoids are associated with mature teratoma. Twenty two metastatic carcinoids $(81.5 \%)$ were from the GI tract, four $(14.8 \%)$ from the pancreas, and one $(3.7 \%)$ from the posterior thorax location. There was significant difference of Ki67 index between primary (median 2.3\%, range, 0.6-8.4\%) and metastatic carcinoid tumors (median 9.7\%, range, $1.3-$ $46.7 \%)(p=0.002)$. The survival time is much shorter among patients with metastatic carcinoid tumor (median survival 5.8 years) comparing to primary ovarian carcinoid tumor (median 14.2 years) $(p=0.0005)$. A strong association between Ki67 index and patient survival time was identified (Hazard ratio for 1-percentage point increase 1.11, $p=0.001$ ). Comparing to primary ovarian carcinoid tumor, metastatic carcinoid usually exhibits a higher Ki67 index and a worse outcome.
\end{abstract}

Keywords Ovarian carcinoid tumor $\cdot$ Proliferation index $\cdot$ Ki67 $\cdot$ Metastasis

\section{Introduction}

Primary ovarian carcinoid tumors are rare neoplasms that constitute about $0.1 \%$ of all ovarian carcinomas and less than $1 \%$ of all carcinoid tumors [1]. This entity was first described by Stewart et al. in 1939 and subsequent studies have demonstrated that a majority of primary ovarian carcinoids occur in association with mature cystic teratoma, with a small number in pure form. Most primary ovarian carcinoid tumors are seen in

Yajue Huang

Huang.Yajue@mayo.edu

1 Department of Laboratory Medicine and Pathology, Mayo Clinic, Rochester, MN, USA

2 Department of Health Sciences Research, Mayo Clinic, Rochester, MN, USA perimenopausal woman with presentation of unilateral ovarian mass. Carcinoid syndrome usually presents in about $30 \%$ of these patients and is generally associated with insular-type growth pattern [2]. Comparing to primary ovarian carcinoid tumors, neuroendocrine tumors from extra ovarian origin with metastasis to the ovary are relatively common. These tumors tend to arise from gastrointestinal origin, which usually involves bilateral ovaries.

Primary and secondary carcinoid tumors of the ovary share similar histological growth pattern but differ from the clinical behavior, pathologic features, and treatments, and prognosis [2]. Cell proliferative rate is an important factor in the determination of neuroendocrine tumor prognosis. As limited data are available regarding tumor cell proliferation index in predicting the physiological features of carcinoid tumors involving the ovary, in this study, we investigate Ki67 expression in both primary and secondary ovarian carcinoid tumors and explore the correlation with patients' clinical outcomes. 


\section{Material and Methods}

\section{Study Subjects}

This study was approved by the institutional review board (IRB) of Mayo Clinic, Rochester, MN. A total of 36 eligible patients with ovarian carcinoid/well-differentiated neuroendocrine tumor diagnosis from 1994 to 2005 were included in this study.

Clinical and pathological information including tumor size, laterality, age (at the time of ovary involvement and at death if available), time interval from primary diagnosis to the metastasis, total follow-up time, and cause of death (if available) were collected through carefully reviewing of clinical notes and pathology reports.

\section{Immunohistochemistry Stains}

Five-micron $(5 \mu \mathrm{m})$ tissue sections were cut from formalinfixed, paraffin-embedded tissue blocks from 36 ovarian carcinoids, including 9 primary and 27 metastatic ovarian carcinoids. Tissue sections were subsequently deparaffinized and rehydrated, and then stained with monoclonal antibody against Ki67 (mouse anti-human Clone MIB-1, DAKO), TTF-1 (mouse anti-human Clone SPT24, Leica), CDX2 (rabbit anti-human Clone EPR2764Y, Cell Marque), and Islet-1 (mouse anti-human Clone 1H9, Abcam) using a Ventana Discovery Ultra IHC stainer. DAB (3, 3'-diaminobenzidine) was used as chromogen. Appropriate positive and negative controls were set up at the same time as IHC performed.

\section{Digital Analysis}

Ki67 immunostained slides were scanned at $\times 40$ magnification on the Aperio ScanScope AT Turbo brightfield instrument (Leica Biosystems) at a resolution of $0.25 \mu \mathrm{m}$ per pixel. The images were 24-bit contiguous standard pyramid-tiled TIFFs compressed via JPEG2000 with a quality setting of
70. For digital image analysis, the Aperio ImageScope Software (Leica Biosystems) was utilized. Fixed-sized tiles were placed on the selected region of interest (Fig. 1). Care was taken to avoid staining artifacts. A nuclear algorithm was used to analyze the selected tissue. Quality control was performed on a subset of cases.

\section{Statistical Analysis}

Ki67 percentage was summarized with medians, interquartile ranges, and ranges and was compared between those with ovarian primary versus metastatic carcinoid tumors with Wilcoxon rank-sum tests. Associations between group and Ki67 with overall survival from time of ovarian involvement were assessed with likelihood ratio tests from a Cox proportional hazards regression model and were summarized with median (Kaplan-Meier estimate) survival and hazard ratios. Ninety-five percent confidence intervals (CI) were also reported. Statistical analyses were performed using SAS version 9.4 or R. $p$ values less than 0.05 were considered statistically significant.

\section{Results}

\section{Clinical-Pathological Characteristics of Patients}

A total of 36 cases including 9 ovarian primary and 27 metastatic carcinoid cases were investigated in the current study. Seven out of nine $(77.8 \%)$ ovarian primary carcinoids were associated with mature teratoma. One patient possessed a remote history of ovary dermoid tumor with a component of carcinoid more than 20 years ago. Twenty two metastatic carcinoids $(81.5 \%)$ were from the gastrointestinal tract, four (14.8\%) from the pancreas, and one $(3.7 \%)$ from the posterior thorax location.

The age incidence of patients with ovarian carcinoids showed a wide range, from 33 to 83 years, and the majority
Fig. 1 A Metastatic NET from the GI tract, Ki67 index 7.7\%. B Metastatic NET from the GI tract, Ki67 index 13.2\%; a scanned image of Ki67 IHC; b fixed-sized tiles on selected region of interest; c fixed-sized tiles on selected region of interest with analysis mark-up
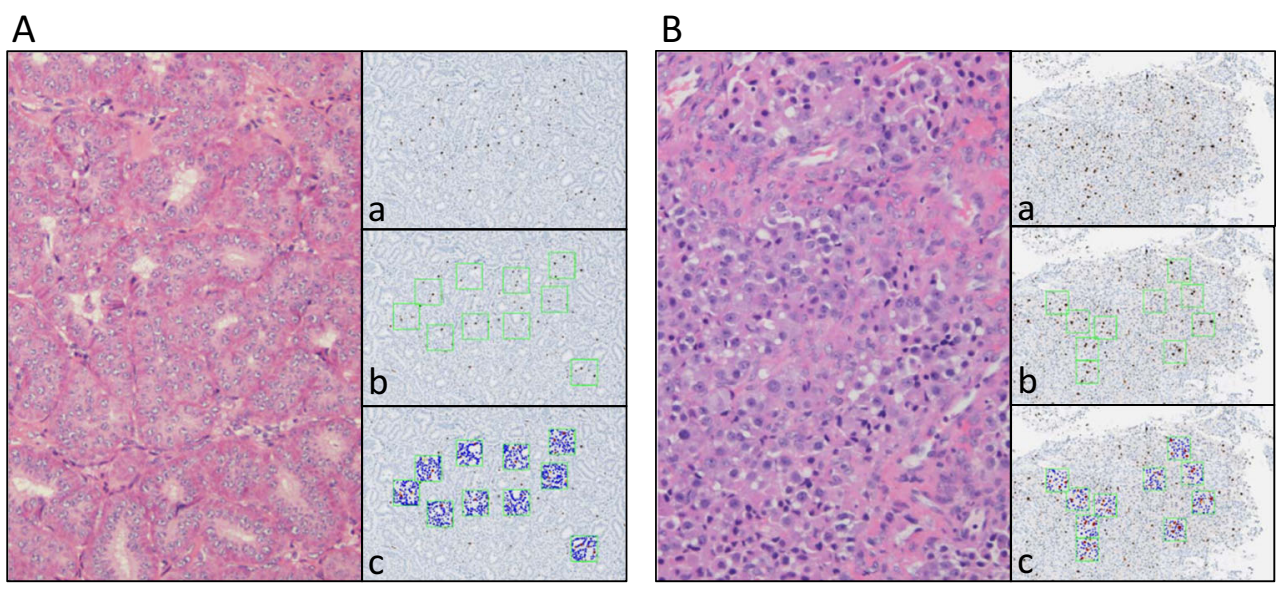
of patients $(77.8 \%)$ were post-menopausal (median age 64 years). We did not find significant age difference between patients with primary and metastatic ovarian carcinoids (Table 1).

Three out of the nine patients $(33.3 \%)$ with primary ovarian carcinoid tumor presented with elevated urine 5-HIAA levels (ranging from 77 to $141 \mathrm{mg} / 24 \mathrm{~h}$ ) at the time of diagnosis. These three patients also developed carcinoid heart disease prior to the time of ovarian tumor diagnosis. One patient presented with liver and lymph node metastasis despite the fact that her ovarian carcinoid tumor was associated with a mature cystic teratoma. The metastatic lesions were diagnosed by imaging study 1 year after oophorectomy and had been stable during subsequent 11-year follow-up. The other two (66.7\%) patients with carcinoid heart disease died from carcinoid tumor. Interestingly, the tumors from the two deceased patients were not associated with ovarian teratoma. Clinical notes and imaging studies were carefully reviewed on these two patients. There was no evidence of metastatic lesions or other primary tumor sites identified before death (follow-up time 14 years for each of the two patients).

One patient presented with slightly elevated urine 5-HIAA $(4.9 \mathrm{mg} / 24 \mathrm{~h})$ at the time of diagnosis. At the time of 3-year follow-up, her 5-HIAA was still elevated $(4.8 \mathrm{mg} / 24 \mathrm{~h})$ but there was no evidence of systemic carcinoid syndrome.

\section{Gross and Microscopic Features of Ovarian Carcinoids}

At the time of diagnosis, all primary ovarian carcinoids showed unilateral ovary involvement. One patient developed bilateral teratoma, but carcinoid tumor was only detected in one of the ovaries. In two cases, the primary ovarian carcinoid tumors were not associated with ovarian teratoma. The average size of the tumors was $8.0 \mathrm{~cm}$ in greatest dimension.

Among metastatic ovarian carcinoid tumors, 9 cases $(33.3 \%)$ showed unilateral involvement and 18 (66.7\%) were bilateral, with a wide size ranges from 2.7 to $17.5 \mathrm{~cm}$. Except one case, all metastatic ovarian carcinoid tumors were preceded or accompanied by extra ovarian metastases, most commonly liver and omentum involvement.

Microscopically, four out of nine $(44.4 \%)$ primary ovarian carcinoids displayed insular growth pattern, with variably sized tumor nests with multiple lumens and simple small glandular formations. Four (44.4\%) cases were strumal carcinoids and one (11.2\%) was mucinous subtype. None of the nine primary ovarian carcinoids demonstrated necrosis. Mitoses were counted in 10 high power fields (HPF). Eight out of nine (88.9\%) primary ovarian carcinoids showed 0-1 mitosis per $10 \mathrm{HPF}$. Only one case showed three mitoses per $10 \mathrm{HPF}$.

As for the metastatic ovarian carcinoid tumors, we only included those that metastasized from primary tumors with diagnoses of either grade 1 or 2 neuroendocrine tumor (NET). No ovarian lesions from higher grade primary NET were included.

\section{Immunohistochemistry Staining Profiles of Primary Ovarian Carcinoids}

Three different markers, TTF-1, CDX2, and Islet-1 IHC, were performed on the nine primary ovarian carcinoid tumors. Three out of four $(75 \%)$ of the strumal carcinoids showed strong and diffuse TTF-1 staining, and one $(25 \%)$ had focal positivity of TTF-1. None of the other five ovarian carcinoids stained positive with TTF-1 antibody. CDX2 showed strong and diffuse staining pattern in six $(66.7 \%)$ cases, focally positive staining in one $(11.1 \%)$ case, and was negative in two $(22.2 \%)$ strumal carcinoid tumors. Islet-1 stained two strumal carcinoid tumors which also expressed CDX2 and TTF-1 (Fig. 2).

\section{Proliferation Index (Ki67) and Survival Data}

Ki67 IHC stains were performed on all cases. Digital analysis was performed with the Aperio ${ }^{\circledR}$ ePathology Solutions software (Leica Biosystems) by cytotechnologists trained in digital image analysis. Figure 1 shows representative images of tumor histology and Ki67 IHC stains.

Ki67 protein was expressed in a range of 0.6 to $8.4 \%$ of tumor cells of primary ovarian carcinoid, with a median of $2.3 \%$. There were more Ki67-positive tumor cells in metastatic tumors, with a range of 1.3 to $46.7 \%$ and median of $9.7 \%$. There was a significant difference of $\mathrm{Ki} 67$ index between primary and metastatic carcinoid tumors $(p=0.002)$. Table 2
Table 1 Clinical feature of ovarian carcinoid tumors

\begin{tabular}{|c|c|c|c|c|c|c|c|}
\hline \multirow[t]{2}{*}{ Primary } & \multirow[t]{2}{*}{$N$} & \multirow{2}{*}{$\begin{array}{l}\text { Age (year) } \\
\text { Median (range) }\end{array}$} & \multicolumn{2}{|c|}{ Association with teratoma } & \multicolumn{3}{|c|}{ Origin of metastasis } \\
\hline & & & Yes & No & GI tract & Pancreas & Unknown*** \\
\hline Ovarian & 9 & $68(33-73)^{*}$ & 7 & 2 & & & \\
\hline Non-ovarian & 27 & $64(36-83)^{* *}$ & & & 22 & 4 & 1 \\
\hline
\end{tabular}

*Age at diagnosis of primary ovarian carcinoid

**Age at ovarian involvement of metastatic carcinoid

***Posterior thorax location 
Fig. 2 A (a) Struma ovarii H\&E, (b) TTF-1 IHC, (c) CDX2 IHC, (d) Islet-1 IHC. B (a) Ovarian carcinoid tumor H\&E, (b) TTF-1 IHC, (c) CDX2 IHC, (d) Islet-1 IHC. Magnification $\times 200$
A

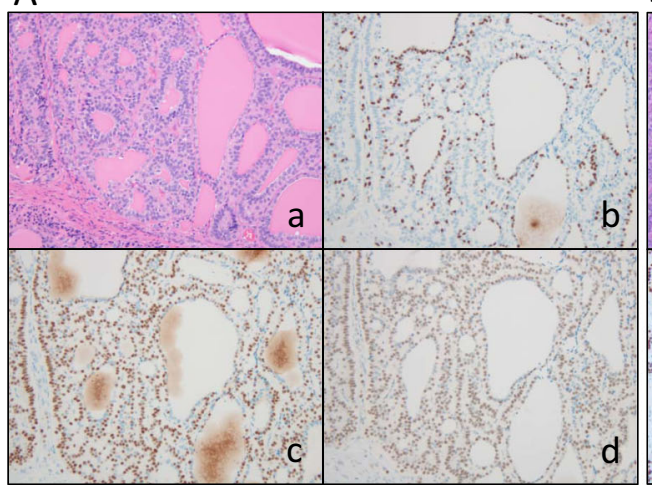

B

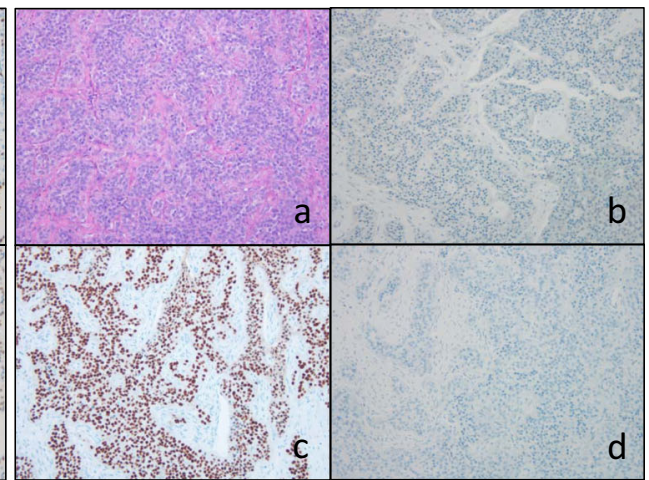

shows Ki67 index in both primary and secondary ovarian carcinoid tumors.

The median survival time was 14.2 years for primary ovarian carcinoid patients (95\% CI 14.0-14.4 years) and 5.8 years of metastatic patients (95\% CI 3.9-13.1 years). The survival time was significantly shorter among patients with metastatic carcinoid tumors $(p=0.0005)$ (Fig. 3A). Cases with Ki67 index $\geq 7.5 \%$ had significantly shorter survival as compared to those $<7.5 \%$ (Hazard ratio $4.43,95 \%$ CI 1.32-20.14, $p=$ 0.01) (Fig. 3B). Considering Ki67 index continuously, the hazard ratio for a 1-percentage point increase is 1.11 (95\% CI $1.04-1.18, p=0.001)$. Regarding the metastatic patients, the effect of Ki67 on overall survival was similar as seen in the full group (HR for 1-percentage point increase $=1.08,95 \% \mathrm{CI}$ $1.009-1.15, p=0.02$ ). There were too few cases to test this effect within the primary ovarian group.

\section{Discussion}

Carcinoid tumors involving the ovary can be either primary or metastatic. Previous studies have shown that primary ovarian carcinoids tend to be confined within the ovary and follow a generally benign course whereas majority of patients with metastatic carcinoids have a low 5-year survival [3, 4]. This is similar to what we found in the current study, in which the median survival time was 14.2 years for primary and 5.8 years for metastatic ovarian carcinoid patients.
Morphologically, primary carcinoid tumors of the ovary are described as insular, trabecular, strumal, and mucinous subtypes [5]. The insular type is the most common histological pattern, followed by the strumal type [6]. The majority of primary ovarian carcinoids occur in association with either cystic teratoma or ovarian epithelial tumors, in which enterochromaffin cells give rise to the carcinoids [7]. However, a small portion of ovarian carcinoids are present in pure form. The origin of these tumors is still unclear, but enterochromaffin cells have been observed within normal ovarian tissue [8]. Studies have shown that insular carcinoids represent tumors of midgut derivation, while trabecular carcinoids are tumors of foregut and hindgut derivation, which may help to explain that only the insular subtype is associated with the carcinoid syndrome. In our study, all four primary carcinoid tumors associated with elevated 5-HIAA, including the one without obvious carcinoid syndrome, are classified as insular subtype.

Most cases of primary ovarian carcinoid tumors are in the early stage and curable by excision alone [9]. Primary ovarian carcinoids only occasionally metastasize with the liver being the most common site. In this study, we had one primary ovarian carcinoid case presenting with liver and lymph node metastasis. Interestingly, the Ki67 stain on the primary tumor showed a very low index $(0.6 \%)$. Thus, primary ovarian carcinoids should be treated as ovarian tumors of low malignant potential.

In primary GI carcinoids, vasoactive substances secreted from tumor go through liver circulation and are rapidly inactivated. By contrast, primary ovarian carcinoid tumors
Table 2 Ki67 expression in ovarian carcinoid tumor

\begin{tabular}{lllll}
\hline $\mathrm{N}$ & $\begin{array}{l}\text { Primary } \\
(N=9)\end{array}$ & $\begin{array}{l}\text { Metastatic } \\
(N=27)\end{array}$ & $\begin{array}{l}\text { Total } \\
(N=36) \\
27\end{array}$ & $\begin{array}{l}p \\
\text { value }\end{array}$ \\
\hline KI67 index & & & & $0.002^{*}$ \\
$\quad$ Median & 2.3 & 9.7 & 7.7 & \\
25th, 75th percentile & $2.2,5.5$ & $5.5,14.5$ & & \\
Range & $(0.6-8.4)$ & $(1.3-46.7)$ & $(0.6-46.7)$ & \\
\hline
\end{tabular}

*Wilcoxon rank sum, primary versus metastatic ovarian carcinoid tumor 
Fig. 3 A Primary and metastatic ovarian carcinoid tumors survival time. B Ki67 index and survival time
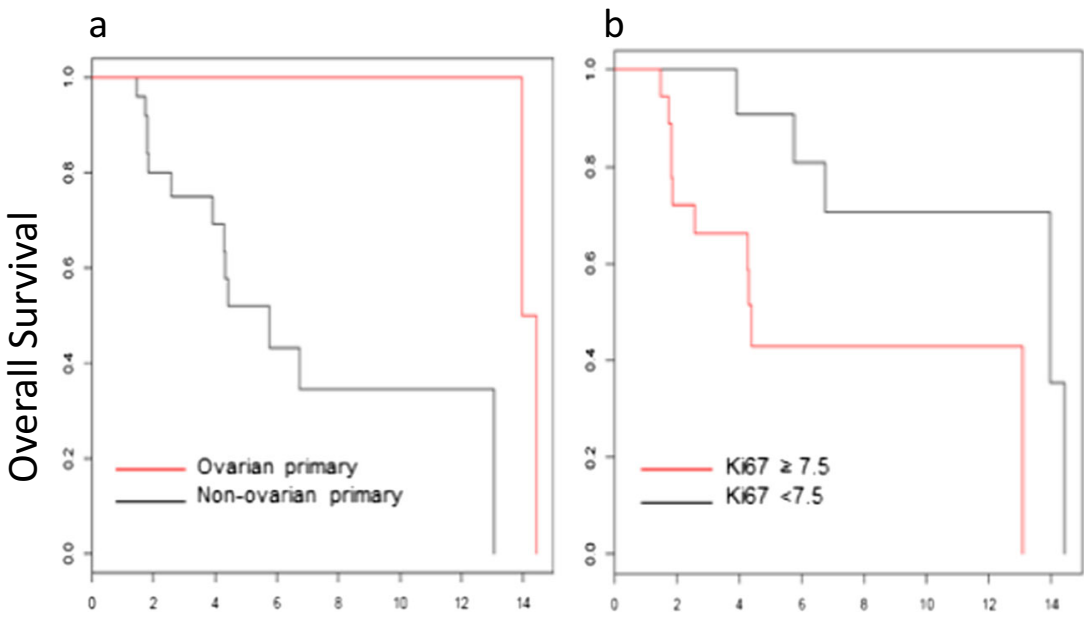

release serotonin or other vasoactive substances directly into the systemic circulation and readily cause carcinoid syndrome. Previous studies have shown different spectrum of neurohormonal peptides expressed by primary ovarian carcinoids comparing to the metastases, which tends to retain the same substances as the original tumor of the midgut [10].

Around $1 / 3$ of patients with primary ovarian carcinoids may develop with carcinoid syndrome with heart disease at an early stage without evidence of metastasis [11]. Generally, the heart failure caused by a carcinoid tumor is characterized by isolated, severe tricuspid regurgitation without significant left-sided valve dysfunction [1]. In our cohort, four patients with primary ovarian carcinoid developed with elevated 5HIAA, but only three patients presented with clinically significant carcinoid syndrome. Among those patients, two died with carcinoid cardiac disease.

The prognosis of stage I primary ovarian carcinoid is with greater than 90\% 5-year survival [3]. In a large study of 329 cases, the authors separated the carcinoids into two groups according to the presence of a dermoid tumor, and their data showed that ovarian carcinoids that are not associated with teratoma generally exhibit larger sizes, higher rate of overall and hepatic metastases, higher rate of carcinoid syndrome, and poorer 5-year survival [12]. In our cohort, two patients died from carcinoid cardiac disease without associated teratoma identified at the time of diagnosis.

The need to distinguish primary from metastatic ovarian carcinoid mainly is due to the dramatic difference of clinical treatment and long-term prognosis. If no prior history was known to the pathologist, differentiation of ovarian carcinoid tumor from metastasis only based on morphology would be difficult [13]. Recently, CDX-2 has been proposed as a useful tool to help with the differential diagnosis [14]. However, other study has shown that CDX-2, as a marker of midgut origin for metastatic carcinoids, also diffusely stains primary ovarian carcinoid with insular and mucinous pattern. Therefore, CDX-2 does not distinguish primary ovarian carcinoids from those that are metastatic from the intestinal tract [15]. In this study, we have shown that the majority of our primary ovarian carcinoid tumors express CDX2; thus, this marker is not helpful in differentiating metastatic ovarian carcinoid from midgut origin. TTF-1, on the other hand, stains strumal carcinoids in the ovary and NET of the site such as the lung therefore is not a sensitive marker for all primary ovarian carcinoids. A possible value of TTF-1 is to show noncarcinoid teratoma components that are initially missed.

In addition to carefully searching for past medical history, there are no immunohistochemical markers which can be used to reliably distinguish between primary and secondary carcinoid tumors involving the ovary. Current pathologic approach is mainly based on association with teratoma or more uncommonly other ovarian neoplasms [16, 17], tumor laterality, and/ or ovarian surface involvement [18]. When none of the above features can be found in a unilateral ovarian low-grade NE tumor and no known primary extra-ovarian carcinoid tumor is identified, a definitive diagnosis can be problematic.

Recently, gene expression profiling has been studied for classifying neuroendocrine tumors of indeterminate primary site. Kerr [19] et al. used a 92-gene cancer classifier investigating 75 NET of various origins. Although primary ovarian carcinoid tumors were not included in this study, the authors found 4 genes with promising discriminatory value for tumor typing and 15 genes for tumor subtyping of gastrointestinal, pulmonary, pancreas, thyroid, or skin origins. In another study, match of maternal isodisomy successfully showed the association between some mucinous carcinomas and ovarian teratomas [20]. These studies provide a promising method that could help with the confirmation of ovarian carcinoid origins. However, as the method is expensive, it has not been proven practical in a clinical setting.

Ki67 labeling index is known to correlate with survival in patients with neuroendocrine tumors of non-ovarian origin [21]. In the current study, we propose that tumor cell proliferation index, defined by tumor cell nuclear Ki67 protein 
expression, can be used to distinguish primary from metastatic ovarian carcinoid. The current study shows a higher proliferation index in metastatic carcinoid tumors comparing to primary ovarian carcinoid. It is worth mentioning that only lowgrade (grades 1 and 2) metastatic NE tumors have been selected in our study, and all high-grade NE carcinomas have been excluded because (1) high-grade metastatic NE carcinoma is rarely confused with ovarian carcinoid, (2) high-grade metastatic NE carcinoma generally has high proliferation rate, and (3) it is well studied that high-grade NE carcinoma possesses a worse prognosis $[22,23]$.

To our knowledge, this is the first study of tumor proliferation rate in comparison of primary ovarian carcinoid tumors versus metastatic carcinoid tumors of the ovaries. Our data indicate that ovarian metastatic carcinoid usually exhibits a high Ki67 index, which can be helpful when differentiation between primary and metastatic carcinoids involving the ovary is needed. Furthermore, Ki67 index can be used as a prognostic parameter as Ki67 index is significantly associated with patient's survival in both primary and metastatic carcinoid tumors.

Open Access This article is distributed under the terms of the Creative Commons Attribution 4.0 International License (http:// creativecommons.org/licenses/by/4.0/), which permits unrestricted use, distribution, and reproduction in any medium, provided you give appropriate credit to the original author(s) and the source, provide a link to the Creative Commons license, and indicate if changes were made.

\section{References}

1. Hong SN, Saric M, Kronzon I. Carcinoid heart disease. J Am Coll Cardiol 2010;55:1996.

2. McGrath S, Nicklin J. Clinicopathological study of ovarian carcinoid tumors. Aust N Z J Obstet Gynaecol 2016;56:508-513.

3. Modlin IM, Lye KD, Kidd M. A 5-decade analysis of 13,715 carcinoid tumors. Cancer 2003;97:934-959.

4. Baker PM, Oliva E, Young RH, et al. Ovarian mucinous carcinoids including some with a carcinomatous component: a report of 17 cases. Am J Surg Pathol 2001;25:557-568.

5. Davis KP, Hartmann LK, Keeney GL, et al. Primary ovarian carcinoid tumors. Gynecol Oncol 1996;61:259-265.

6. A. Talerman. Carcinoid tumors of the ovary. J Cancer Res Oncol 1984;107:125-135.
7. Howitt BE, Kelly P, McCluggage WG. Pathology of Neuroendocrine Tumours of the Female Genital Tract. Curr Oncol Rep 2017;19:59.

8. Hidvegi D, Cibils LA, Sorensen K. et al. Ultrastructural and histochemical observations of neuroendocrine granules in nonneoplastic ovaries. Am J Obstet Gynecol 1982;143:590-594.

9. Gardner GJ, Reidy-Lagunes D, Gehrig PA. Neuroendocrine tumors of the gynecologic tract: A Society of Gynecologic Oncology (SGO) clinical document. Gynecol Oncol 2011;122:190-198.

10. Sporrong B, Falkmer S, Robboy SJ, et al. Neurohormonal peptides in ovarian carcinoids: an immunohistochemical study of 81 primary carcinoids and of intraovarian metastases from six mid-gut carcinoids. Cancer 1982;49:68-74.

11. Moerman VM, Dewilde D, Hermans K. Carcinoid heart disease: typical findings on echocardiography and cardiac magnetic resonance. Acta Cardiol 2012;67:245-248.

12. Soga J, Osaka M, Yakuwa Y. Carcinoids of the ovary: an analysis of 329 reported cases. J Exp Clin Cancer Res 2000; 19:271-280.

13. Rqbboy SJ, Scully RE, Norris HJ. Carcinoid metastatic to the ovary. A clinicopathologic analysis of 35 cases. Cancer 1974; 33:798-781.

14. Desouki MM, Lioyd J, Xu H, et al. CDX2 may be a useful marker to distinguish primary ovarian carcinoid from gastrointestinal metastatic carcinoids to the ovary. Hum Pathol 2013; 44:2536-2541.

15. Rabban JT, Lerwill MF, McCluggage WG, et al. Primary ovarian carcinoid tumors may express CDX-2: a potential pitfall in distinction from metastatic intestinal carcinoid tumors involving the ovary. Int J Gynecol Pathol 2009; 28:41-48.

16. Bohara S, Agarwal S, Khuraijam B, et al. Strumal carcinoid ovary with mucinous cystadenoma presenting as a large abdominal mass and increased tumour marker level. J Obstet Gynaecol 2013; 33: 637-639.

17. Delić R. Mucinous cystadenoma of the ovary with carcinoid tumour in a 23-year-old nulliparous woman. J Obstet Gynaecol 2017; 37:543-544.

18. Strosberg J, Nasir A, Cragun J, et al. Metastatic carcinoid tumor to the ovary: a clinicopathologic analysis of seventeen cases. Gynecol Oncol 2007; 106:65-68.

19. Kerr SE, Schnabel CA, Sullivan PS et al. A 92-gene cancer classifier predicts the site of origin for neuroendocrine tumors. Mod Pathol. 2014; 27:44-54.

20. Kerr SE, Flotte AB, McFalls MJ, et al. Matching maternal isodisomy in mucinous carcinomas and associated ovarian teratomas provides evidence of germ cell derivation for some mucinous ovarian tumors. Am J Surg Pathol. 2013; 37:1229-1235.

21. Yang Z, Tang LH, Klimstra DS. Effect of tumor heterogeneity on the assessment of Ki67 labeling index in well-differentiated neuroendocrine tumors metastatic to the liver: implications for prognostic stratification. Am J Surg Pathol 2011; 35:853-860.

22. Conte B, George B, Overman M, et al. High-Grade Neuroendocrine Colorectal Carcinomas: A Retrospective Study of 100 Patients. Clin Colorectal. Cancer 2016; 15:e1-e7.

23. Deep NL, Ekbom DC, Hinni ML, et al. High-Grade Neuroendocrine Carcinoma of the Larynx: The Mayo Clinic Experience. Ann Otol Rhinol Laryngol 2016; 125:464- 469. 